\title{
Stabilization and control of topological magnetic solitons via magnetic nanopatterning of exchange bias systems
}

\author{
Edoardo Albisetti ${ }^{1,2, a)}$, Annalisa Calò', Martin Spieser ${ }^{3}$, Armin W. Knoll ${ }^{4}$, \\ Elisa Riedo ${ }^{1,5}$, Daniela Petti ${ }^{2}$
}

${ }^{1}$ Advanced Science Research Center, CUNY Graduate Center, 85 St. Nicholas Terrace, New York, New York 10031, USA.

${ }^{2}$ Dipartimento di Fisica, Politecnico di Milano, 20133 Milano, Italy.

${ }^{3}$ SwissLitho AG, 8805 Zürich, Switzerland.

${ }^{4}$ IBM Research-Zurich, 8803 Rüschlikon, Switzerland.

${ }^{5}$ Tandon School of Engineering, New York University (NYU), 6 MetroTech Center

Brooklyn, NY 11201, New York, USA.

a) Correspondence to: edoardo.albisetti@polimi.it; 
Stabilizing and manipulating topological magnetic quasiparticles in thin films is of great interest for potential applications in data storage and information processing. Here, we present a strategy for stabilizing magnetic vortices and Bloch lines with controlled position, vorticity and chirality in a continuous exchange bias system. By tailoring vectorially the unidirectional anisotropy of the system at the nanoscale, via thermally assisted magnetic scanning probe lithography (tam-SPL), we show experimentally and via micromagnetic simulations the nonvolatile creation of vortex-antivortex pairs. In addition, we demonstrate the deterministic stabilization of cross and circular Bloch lines within patterned Néel magnetic domain walls. This work enables the implementation of complex functionalities based on the control of tailored topological spin-textures in spintronic and magnonic nanodevices.

Topologically protected magnetic spin-textures, such as magnetic vortices, Bloch lines, and skyrmions, are increasingly attracting attention for their applications in a wide variety of fields, ranging from data storage and processing ${ }^{1-5}$, to medicine ${ }^{6}$. For example, in racetrack memories ${ }^{7-9}$, the information is stored by means of magnetic quasiparticles which are controllably stabilized and manipulated via external stimuli within nanowire-based spintronic devices. The dynamic properties of vortices, i.e. their excitation, the core reversal, and their coupling ${ }^{10-14}$ have been extensively studied and are at the basis, for example, of magnetic vortex oscillators ${ }^{15-17}$. Furthermore, the spin-texture topology has a crucial role in the propagation of domain walls ${ }^{18,19}$. The interaction of vortices with spin-waves have been investigated $\mathrm{d}^{20-22}$ and in particular, recently, the use of vortex cores as emitters of short-wavelength spin-waves was demonstrated ${ }^{23}$.

For all these applications, the development of methods for stabilizing and manipulating topological solitons is of capital importance. Among these methods, patterning ferromagnetic micro and nanostructures such as disks or ellipses is widely employed, and it allows the stabilization of vortices (with topological winding number +1 ) by exploiting the shape anisotropy of the system, which favors the circulation of the magnetization, for removing magnetostatic charges at the disk edges ${ }^{24,25}$. The injection of a spin-polarized current in the nanocontact geometry is a well-established method for nucleating vortex-states in a continuous film through spin-transfer torque ${ }^{26}$. The controlled stabilization of antivortex ground states (with topological winding number -1), on the other hand, is more challenging and is usually achieved as a result of the stabilization of multiple vortices in a vortex-antivortex (V-A) array ${ }^{17,27-29}$.

Nevertheless, positioning and manipulating single topological spin-textures with deterministically controlled position, vorticity and chirality, and in a non-volatile way, is extremely challenging. In this work, we demonstrate a straightforward strategy for stabilizing and manipulating with nanoscale 
precision magnetic topological solitons, by reversibly patterning the unidirectional anisotropy of a continuous exchange bias system via thermally assisted magnetic scanning probe lithography (tamSPL) $)^{30-33}$. In particular, we show experimentally and with micromagnetic simulations, the nonvolatile stabilization and manipulation of vortices with tailored vorticity and chirality, and the nucleation of single Bloch lines within patterned Néel domain walls. Furthermore, we show that Bloch lines stabilized within patterned arbitrarily-shaped domain walls can be controllably displaced along the wall via static external fields.

The studied systems consist in $\operatorname{Ir}_{22} \mathrm{Mn}_{78}$ 7-10/Co40Fe $40 \mathrm{~B}_{20}$ 5-20 /Ru 2 (thicknesses in nm) multilayers grown via magnetron sputtering as reported in Ref. 30, featuring an exchange bias field ranging from 2.5 to $9 \mathrm{mT}$, depending on the antiferromagnetic and ferromagnetic film thicknesses. Tam-SPL was performed via NanoFrazor Explore (SwissLitho AG, Zurich), a commercial thermal scanning probe lithography system, using a cantilever design (See Supplementary Note 1) that provides higher tip heater temperatures compared to the previous design ${ }^{34}$ at temperatures higher than the silicon inversion temperature. For patterning, the thermal probe was swept on the sample surface in a rasterscan fashion, and heated via joule effect by applying voltage pulses across the heater region of the cantilever. The heating time was $40 \mu \mathrm{s} /$ pixel, with a $10 \mathrm{~nm}$ x $10 \mathrm{~nm}$ pixel size and a $100 \mu \mathrm{s} / \mathrm{pixel}$ pixel time. For patterning regions with different exchange bias direction, the orientation of the external magnetic field during the localized field cooling was controlled by a rotatable permanent magnet which generated a uniform 600 Oe magnetic field directed within the plane of the sample. Micromagnetic simulations of the ground state of the exchange bias system were carried out by solving the Landau-Lifshitz-Gilbert equation, using the software MuMax335. The total simulated volume had dimensions $5120 \times 5120 \times 5 \mathrm{~nm}^{3}$ with a cell size of $5 \times 5 \times 5 \mathrm{~nm}^{3}$, and a periodic boundary condition in the $x$-direction. For the CoFeB film, the saturation magnetization was $M_{\mathrm{s}}=1140 \mathrm{kA} / \mathrm{m}$, exchange constant was $A_{\mathrm{ex}}=1.2 \cdot 10^{-11} \mathrm{~J} / \mathrm{m}$. The Gilbert damping parameter was set to $\alpha=0.5$. The exchange bias field was modeled as an external magnetic field with different in-plane direction and strength, as indicated.

In Figure 1 we demonstrate the controlled nucleation of vortex-antivortex pairs in a CoFeB 5/IrMn 7/Ru 2 (dimensions in $\mathrm{nm}$ ) exchange bias system. The patterned exchange bias field configuration (panels (a), (d)), the magnetic force microscopy image (panels (b), (e)), and the results of micromagnetic simulations (panels (c), (f)) are presented. Blue arrows indicate the direction of the patterned exchange bias field, black arrows the direction of the magnetization. In the micromagnetic simulation, the red and blue colors indicate the divergence of the magnetization, which is related to the experimental MFM contrast. In panels (a-c), we show that a vortex-antivortex pair is stabilized by patterning sequentially 4 triangular-shaped areas with unidirectional anisotropy oriented in a 
Publishing unterclockwise direction. Outside the patterned area, the unidirectional anisotropy is uniformly uirected along $-x$. Such a non-uniform configuration of the exchange bias field, which in turn stabilized a complex non-uniform magnetization configuration, generates a local potential minimum for the vortex core in the center of the patterned region (pink circle in panels a, d), and an antivortex core at the boundary of the patterned region (indicated with a yellow circle). The MFM image and micromagnetic simulation of the pattern at remanence confirm the stabilization of two 90 deg domain walls on the lateral sides of the square pattern, and one 180 deg wall on the bottom side. It is worth noting that the 180 deg Néel wall, marked by a yellow dashed line in the sketch, constitutes equilibrium positions for the antivortex core, which can be displaced from its initial position via external fields, as it will be discussed in the following. We verified the stability of the patterned magnetic configuration over time by remeasuring the patterns after 8 months (see also Ref 30). We remark that the process of field cooling and the exchange bias are also exploited for memory applications ${ }^{36}$, and magnetic sensing ${ }^{37}$, e.g. for pinning the magnetization of the reference electrode in magnetic tunnel junctions and spin-valves. The properties of the vortex-antivortex pairs can be tailored by controlling the direction of the patterned exchange bias field with respect to the geometrical shape of the patterned area. In particular, as shown in panels (d-f), by changing the direction of the patterned exchange bias field, the chirality of the vortex and the location of the antivortex can be controlled. Indeed, the chirality of the vortex is counterclockwise, as the one of the patterned exchange bias field, and the locations of both the antivortex and of the 180 deg Néel wall shifts to the top side of the patterned area.

The strength of the patterned exchange bias field determines both the pinning strength of the vortex core and the stability of the vortex-antivortex pair against annihilation, driven by the vortexantivortex attraction. These two aspects are studied via micromagnetic simulations, as shown in Figure 2. In panel a), the black line and circles show the minimum exchange bias field strength $H_{\mathrm{eb}}$ needed for stabilizing the vortex-antivortex pair, as a function of the lateral size of the square pattern $L$. We observe that, by increasing the size of the patterned area from $0.125 \mu \mathrm{m}$ to $1 \mu \mathrm{m}$, the minimum exchange bias field required decreases from $55 \mathrm{mT}$ down to $2.5 \mathrm{mT}$. Such trend reflects the fact that by increasing the pattern size, also does the distance between the equilibrium positions of the vortex and antivortex, which in turn leads to a weaker attractive force that can be countered by weaker exchange bias fields. The red curve and squares show the corresponding distance $A$ between the vortex and antivortex, ranging from $50 \mathrm{~nm}$ to $450 \mathrm{~nm}$. As shown schematically in the inset, the equilibrium positions do not represent the geometrical center, for the vortex, and corner, for the antivortex, due to the attractive force between the vortex and antivortex. In panel b), we study via micromagnetic simulations the displacement $D$ of the vortex core from its equilibrium position as a 
gunction of small external applied fields, for systems characterized by different exchange bias fields. The simulated pattern size is a $5 \mu \mathrm{m}$ square, therefore the vortex core equilibrium position is located in the geometrical center of the pattern due to the large distance between vortex and antivortex. The inset displays the micromagnetic configuration of the system for $H_{\mathrm{eb}}=6 \mathrm{mT}$ and $H_{\mathrm{ext}}=5 \mathrm{mT}$, showing the vortex core and the 90 deg Néel domain walls displacement with respect to the initial equilibrium position. As expected, we observe that the core displacement induced by the external field decreases for increasing exchange bias fields ranging from $3 \mathrm{mT}$ up to $18 \mathrm{mT}$, due to the increased pinning strength associated with the unidirectional anisotropy.

In the following, we demonstrate that it is possible to deterministically stabilize either vortex or antivortex Bloch lines (also named circular and cross Bloch lines, respectively) with tailored chirality within patterned Néel domain walls, by properly designing the boundary conditions at the domain wall edges. Furthermore, we show that the patterned domain wall constitutes a nanoscale racetrack for Bloch lines, which can be controlled and steered along the wall via external fields.

In Figure 3(a-c) we show experimentally and with simulations, the stabilization of a vortex Bloch line within a patterned 180 deg Néel wall, in a CoFeB 20 / IrMn 10 / Ru 2 (thicknesses in nm) system. In panel (a), a sketch of the configuration of the patterned exchange bias is shown. The 180 deg wall is defined by rectangular regions with magnetization oriented in opposite directions along $+y$ and $-y$. In such a system, the orientation of the magnetization within the domain wall, i.e. the chirality of the domain wall, is determined by the boundary conditions, hence by the direction of the magnetization at the edges of the domain wall. The Bloch line is stabilized by imposing two opposite chiralities at the two ends of the wall, by defining two regions with oppositely oriented magnetization. It is worth noting, that both the chirality of the Bloch lines, and their vorticity, i.e. vortex or antivortex, can be tailored by controlling the orientation of the magnetization of the central domains. In particular, in panel (b), we show the MFM image of a vortex Bloch line with clockwise chirality within a straight 180 deg Néel wall. The magnetization within the wall points towards $-x(+x)$ in the bottom (top) part of the wall, as indicated by the opposite MFM contrast, in good agreement with the micromagnetic simulations of panel (c). The bright/dark colors at the top and bottom sides of the MFM image confirm the opposite direction of the magnetization in the two domains. As shown in panel (e), (f) an antivortex Bloch line is stabilized by patterning an opposite magnetization direction in the two central domains (as sketched in panel (d)).

In Figure 4, we show, via micromagnetic simulations, that Bloch lines stabilized via tam-SPL can be manipulated within the nanopatterned domain walls via static external magnetic fields, without altering the magnetic configuration of the domain wall at remanence. In order to study the Bloch lines motion, a damping parameter $\alpha=0.02$ was used for these simulations ${ }^{38}$. In panel a), an antivortex 
Bloch line is stabilized at the top edge of a straight 180 deg wall (see sketch on the left), by applying an initialization field $H_{\text {init }}$ along $-x$. The exchange bias field strength was $12 \mathrm{mT}$. The central panels show the $x$-component of the magnetization, which allows to clearly locate the Bloch line within the wall, as a function of time. From left to right, we show that, by applying a $6.7 \mathrm{mT}$ static external field along $+x$, the Bloch line can be displaced from the top edge down to the bottom edge of the wall (see also Supplementary Note 3). During the displacement, the Bloch line passes through metastable states, as shown in Supplementary Note 2 and Fig. S2. In panel b), a vortex Bloch line is stabilized on the top edge of a zig-zag 180 deg wall. The shape of the wall is determined by the geometry of the patterned central domains, as shown in the sketch on the left. The Bloch line is displaced from its initial position, and, interestingly, it follows the complex profile of the wall and stabilizes in proximity of the lower domain, demonstrating the possibility of continuously manipulating Bloch lines along arbitrarily shaped tracks. Crucially for applications, the position and shape of the domain wall at remanence, which is determined by the patterned exchange bias profile, is not affected by the field applied for the Bloch line displacement (see Supplementary Note 2 and Fig. S2).

In conclusion, we presented a strategy for the reversible, non-volatile stabilization and nanoscale manipulation of magnetic vortices and Bloch lines, within continuous exchange bias systems. We showed that, by vectorially nanopatterning the unidirectional magnetic anisotropy, it is possible to stabilize vortices with controlled position, vorticity and chirality, and to deterministically stabilize cross and circular Bloch lines within patterned arbitrarily shaped Néel domain walls. Finally, we showed that such domain walls can be employed as reconfigurable conduits where the Bloch lines are manipulated and displaced via static external fields. Our work opens the way towards the study and integration of functionalities based on tailored topological spin-textures in next-generation spintronic and spin-wave devices, such as finely tuned spin-wave emitters/detectors and magnetic oscillators based on arrays of coupled vortices.

\section{References}

1 T.H. Kim, S. Cheon, and H.W. Yeom, Nat. Phys. 13, 444 (2017).

${ }^{2}$ G. Finocchio, F. Büttner, R. Tomasello, M. Carpentieri, and M. Kläui, J. Phys. D. Appl. Phys. 49, 423001(2016).

${ }^{3}$ G. Hrkac, P.S. Keatley, M.T. Bryan, and K. Butler, J. Phys. D. Appl. Phys. 48, 453001 (2015).

${ }^{4}$ S. Zhang, A.A. Baker, S. Komineas, and T. Hesjedal, Sci. Rep. 5, 15773 (2015).

${ }^{5}$ D. Kumar, S. Barman, and A. Barman, Sci. Rep. 4, 4108 (2015).

${ }^{6}$ D.-H. Kim, E. a Rozhkova, I. V Ulasov, S.D. Bader, T. Rajh, M.S. Lesniak, and V. Novosad, Nat. Mater. 9, 165 (2010). 
7 S.S.P. Parkin, M. Hayashi, and L. Thomas, Science 320, 190 (2008).

A. Fert, V. Cros, and J. Sampaio, Nat. Nanotechnol. 8, 152 (2013).

${ }^{9}$ L.D. Geng and Y.M. Jin, J. Magn. Magn. Mater. 423, 84 (2017).

${ }^{10}$ S. Kaka, M.R. Pufall, W.H. Rippard, T.J. Silva, S.E. Russek, and J.A. Katine, Nature 437, 389 (2005).

${ }^{11}$ B. Van Waeyenberge, A. Puzic, H. Stoll, K.W. Chou, T. Tyliszczak, R. Hertel, M. Fähnle, H. Brückl, K. Rott, G. Reiss, I. Neudecker, D. Weiss, C.H. Back, and G. Schütz, Nature 444, 461 (2006).

${ }^{12}$ K. Yamada, S. Kasai, Y. Nakatani, K. Kobayashi, H. Kohno, A. Thiaville, and T. Ono, Nat. Mater. 6, 270 (2007).

${ }^{13}$ M. Manfrini, J. Von Kim, S. Petit-Watelot, W. Van Roy, L. Lagae, C. Chappert, and T. Devolder, Nat. Nanotechnol. 9, 121 (2014).

${ }^{14}$ S.B. Choe, Y. Acremann, A. Scholl, A. Bauer, A. Doran, J. Stöhr, and H.A. Padmore, Science 304, 420 (2004).

${ }^{15}$ V.S. Pribiag, I.N. Krivorotov, G.D. Fuchs, P.M. Braganca, O. Ozatay, J.C. Sankey, D.C. Ralph, and R.A. Buhrman, Nat. Phys. 3, 498 (2007).

${ }^{16}$ Q. Mistral, M. van Kampen, G. Hrkac, J.-V. Kim, T. Devolder, P. Crozat, C. Chappert, L. Lagae, and T. Schrefl, Phys. Rev. Lett. 100, 257201 (2008).

${ }^{17}$ A. Ruotolo, V. Cros, B. Georges, A. Dussaux, J. Grollier, C. Deranlot, R. Guillemet, K.

Bouzehouane, S. Fusil, and A. Fert, Nat. Nanotechnol. 4, 528 (2009).

${ }^{18}$ Y. Yoshimura, K.-J. Kim, T. Taniguchi, T. Tono, K. Ueda, R. Hiramatsu, T. Moriyama, K.

Yamada, Y. Nakatani, and T. Ono, Nat. Phys. 12, 157 (2015).

${ }^{19}$ S. Emori, U. Bauer, S.-M. Ahn, E. Martinez, and G.S.D. Beach, Nat. Mater. 12, 611 (2013).

${ }^{20}$ J.P. Park and P.A. Crowell, Phys. Rev. Lett. 95, 167201 (2005).

${ }^{21}$ M. Kammerer, M. Weigand, M. Curcic, M. Noske, M. Sproll, A. Vansteenkiste, B. Van Waeyenberge, H. Stoll, G. Woltersdorf, C.H. Back, and G. Schuetz, Nat. Commun. 2, 279 (2011).

${ }^{22}$ M.-W. Yoo, J. Lee, and S.-K. Kim, Appl. Phys. Lett. 100, 172413 (2012).

${ }^{23}$ S. Wintz, V. Tiberkevich, M. Weigand, J. Raabe, J. Lindner, A. Erbe, A. Slavin, and J. Fassbender, Nat. Nanotechnol. 11, 948 (2016).

${ }^{24}$ T. Shinjo, T. Okuno, R. Hassdorf, K. Shigeto, and T. Ono, Science 289, 930 (2000).

${ }^{25}$ K.S. Buchanan, P.E. Roy, M. Grimsditch, F.Y. Fradin, K.Y. Guslienko, S.D. Bader, and V. Novosad, Nat. Phys. 1, 172 (2005).

${ }^{26}$ T. Devolder, J. Von Kim, M. Manfrini, W. Van Roy, L. Lagae, and C. Chappert, Appl. Phys. Lett. 97, 19 (2010). 
J. Li, A. Tan, K.W. Moon, A. Doran, M.A. Marcus, A.T. Young, E. Arenholz, S. Ma, R.F. Yang, C. Hwang, and Z.Q. Qiu, Appl. Phys. Lett. 104, 262409 (2014).

${ }^{28}$ Y. Gaididei, O.M. Volkov, V.P. Kravchuk, and D.D. Sheka, Phys. Rev. B 86, 144401 (2012).

${ }^{29}$ K. Kuepper, M. Buess, J. Raabe, C. Quitmann, and J. Fassbender, Phys. Rev. Lett. 99, 167202 (2007).

${ }^{30}$ E. Albisetti, D. Petti, M. Pancaldi, M. Madami, S. Tacchi, J. Curtis, W.P. King, A. Papp, G.

Csaba, W. Porod, P. Vavassori, E. Riedo, and R. Bertacco, Nat. Nanotechnol. 11, 545 (2016).

${ }^{31}$ E. Albisetti, D. Petti, M. Madami, S. Tacchi, P. Vavassori, E. Riedo, and R. Bertacco, AIP Adv.

7, 55601 (2017).

${ }^{32}$ E. Albisetti, D. Petti, G. Sala, R. Silvani, S. Tacchi, S. Finizio, S. Wintz, A. Caló, X. Zheng, J.

Raabe, E. Riedo, and R. Bertacco, arXiv Prepr. 1712.08293 (2017).

${ }^{33}$ E. Albisetti and D. Petti, J. Magn. Magn. Mater. 400, 230 (2016).

${ }^{34}$ M. Spieser, C. Rawlings, E. Lörtscher, U. Duerig, and A.W. Knoll, J. Appl. Phys. 121, 174503 (2017).

${ }^{35}$ A. Vansteenkiste, J. Leliaert, M. Dvornik, M. Helsen, F. Garcia-Sanchez, and B. Van

Waeyenberge, AIP Adv. 4, 107133 (2014).

${ }^{36}$ D. Petti, E. Albisetti, H. Reichlová, J. Gazquez, M. Varela, M. Molina-Ruiz, A.F. Lopeandía, K.

Olejník, V. Novák, I. Fina, B. Dkhil, J. Hayakawa, X. Marti, J. Wunderlich, T. Jungwirth, and R.

Bertacco, Appl. Phys. Lett. 102, 192404 (2013).

${ }^{37}$ P.P. Sharma, E. Albisetti, M. Massetti, M. Scolari, C. La Torre, M. Monticelli, M. Leone, F. Damin, G. Gervasoni, G. Ferrari, F. Salice, E. Cerquaglia, G. Falduti, M. Cretich, E. Marchisio, M. Chiari, M. Sampietro, D. Petti, and R. Bertacco, Sensors Actuators B Chem. 242, 280 (2017).

${ }^{38}$ M.C. Weber, H. Nembach, B. Hillebrands, M.J. Carey, and J. Fassbender, J. Appl. Phys. 99, 08J308 (2006).

\section{Supplementary Materials}

See supplementary material for details on the high-temperature cantilever design, and for the results of additional micromagnetic simulations on the propagation of Bloch lines along nanopatterned domain walls.

\section{Acknowledgements}

The authors thank Prof. Riccardo Bertacco and Prof. Hans Hug for the valuable discussions. The research leading to these results has received funding from the European Union's Horizon 2020 research and innovation programme under grant agreement no. 705326, project SWING. We 
Publishinģ knowledge the support from the Office of Basic Energy Sciences of the US Department of Energy (grant no. DE-SC0016204), the US Army Research Office and the US National Science Foundation (NSF). This work was partially performed at Polifab, the micro- and nano-technology center of the Politecnico di Milano.

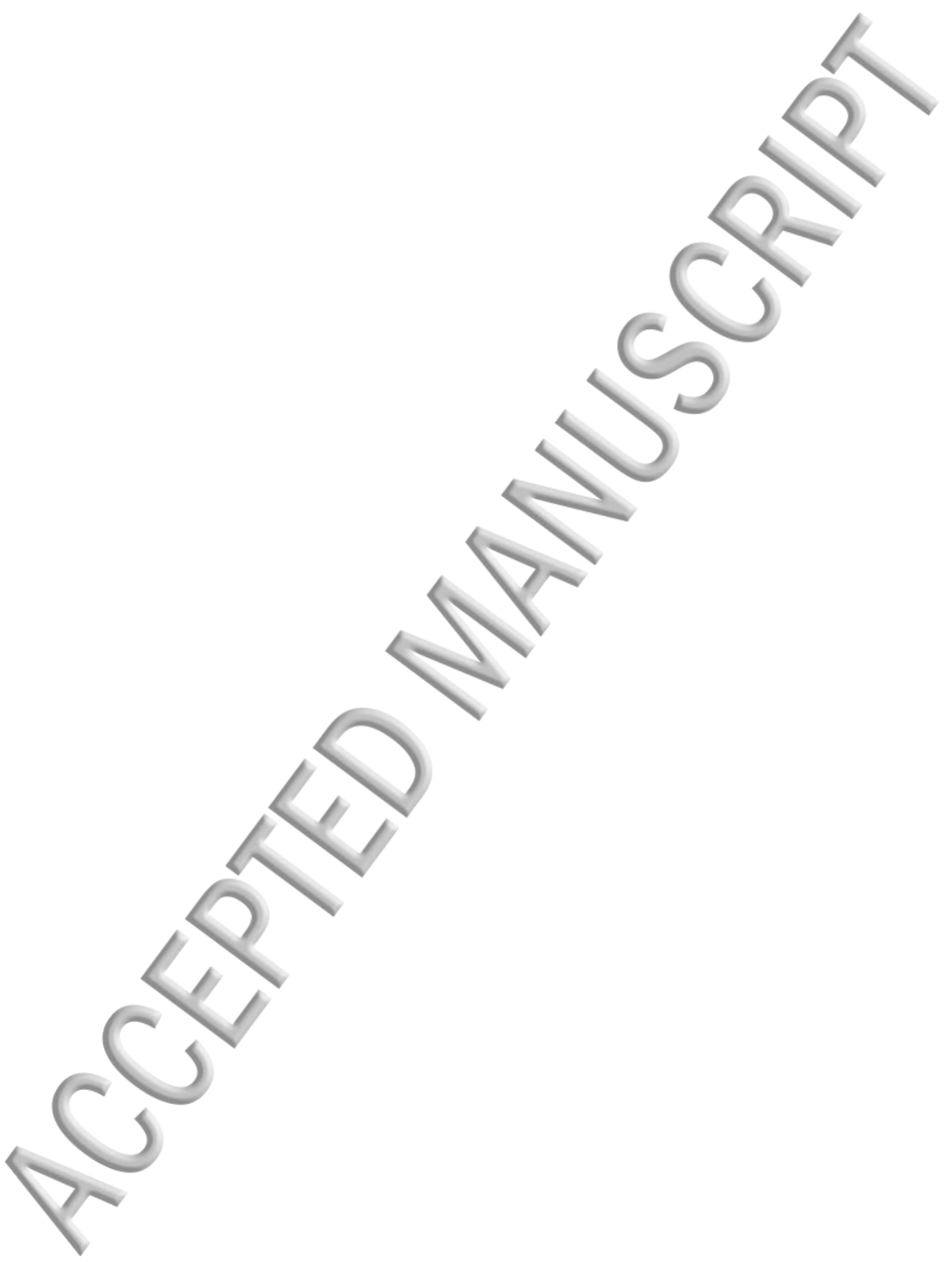




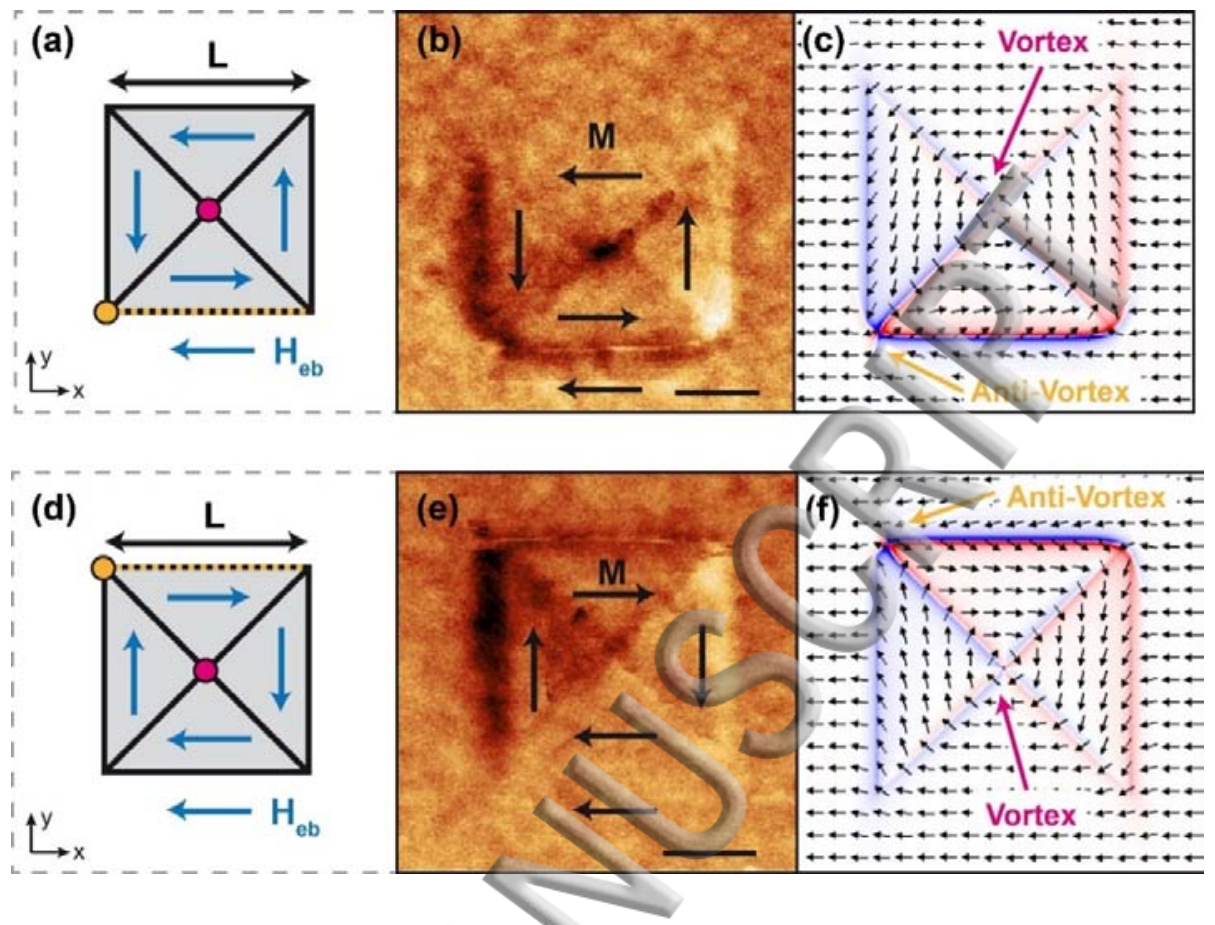

Figure 1. Stabilization of vortex-antivortex pairs. (a), (d) Schematics showing the configuration of the exchange bias patterned via tam-SPL in the IrMn/CoFeB system, for counterclockwise and clockwise chirality, respectively. The direction of the patterned exchange bias is indicated by the blue arrow for each area. $L$ marks the lateral size of the pattern. The pink (orange) circles mark the position of the vortex core (antivortex) within the pattern. The orange dotted line marks the 180 deg Néel domain wall. (b), (e) MFM images of the patterned structure for counterclockwise and clockwise orientation, respectively. The direction of the magnetization is indicated by the black arrows. Scale bar $2 \mu \mathrm{m}$. (c), (f) Corresponding simulated micromagnetic configuration showing the location of the vortex and antivortex. The black arrows indicate the direction of the spins, and the red/blue color marks div (M), which is related to the measured MFM contrast. 

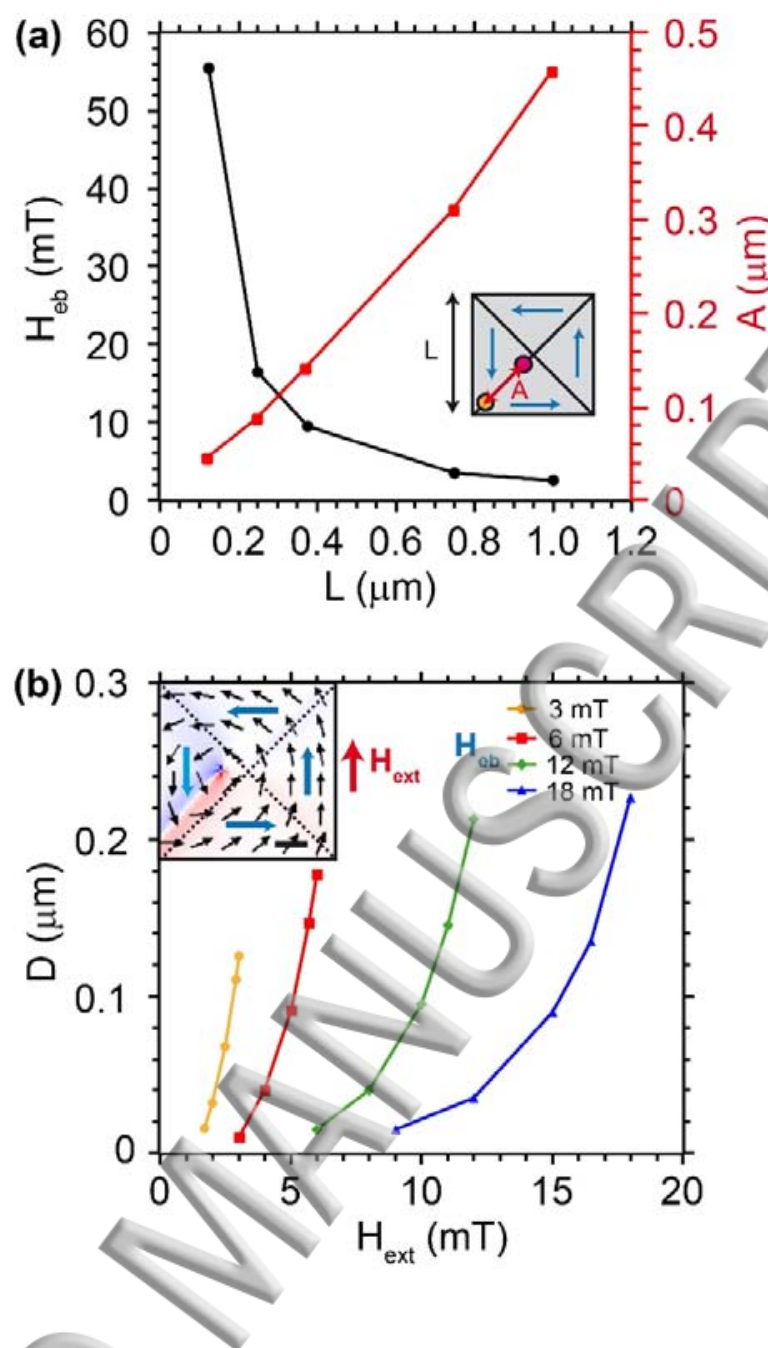

Figure 2. (a) Results of micromagnetic simulations showing the minimum exchange bias field strength $H_{\mathrm{eb}}$ needed for stabilizing vortex-antivortex pairs in the configuration shown in the inset, as a function of the lateral size of the pattern $L$. In red, the corresponding distance $A$ between vortex and antivortex. (b) Distance $D$ between the vortex core and the geometrical center of the pattern, as a function of the external magnetic field $H_{\text {ext }}$, for different exchange bias field strength ranging from 3 $\mathrm{mT}$ up to $18 \mathrm{mT}$. In the inset, micromagnetic simulation showing the vortex core displacement and the orientation of the external magnetic field. Scale bar $100 \mathrm{~nm}$. 
(a)

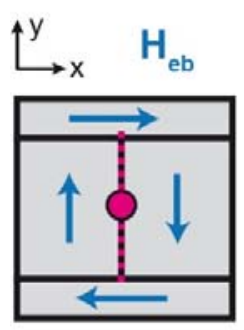

(d)

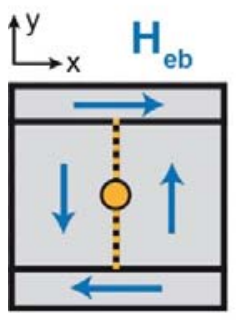

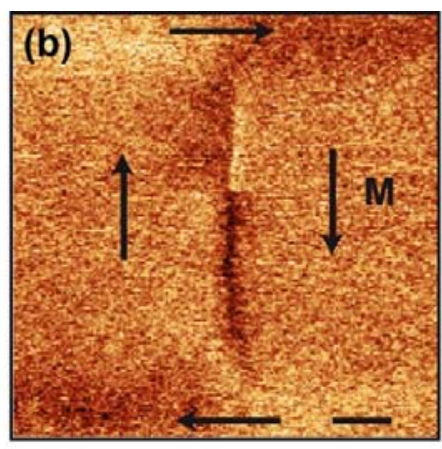
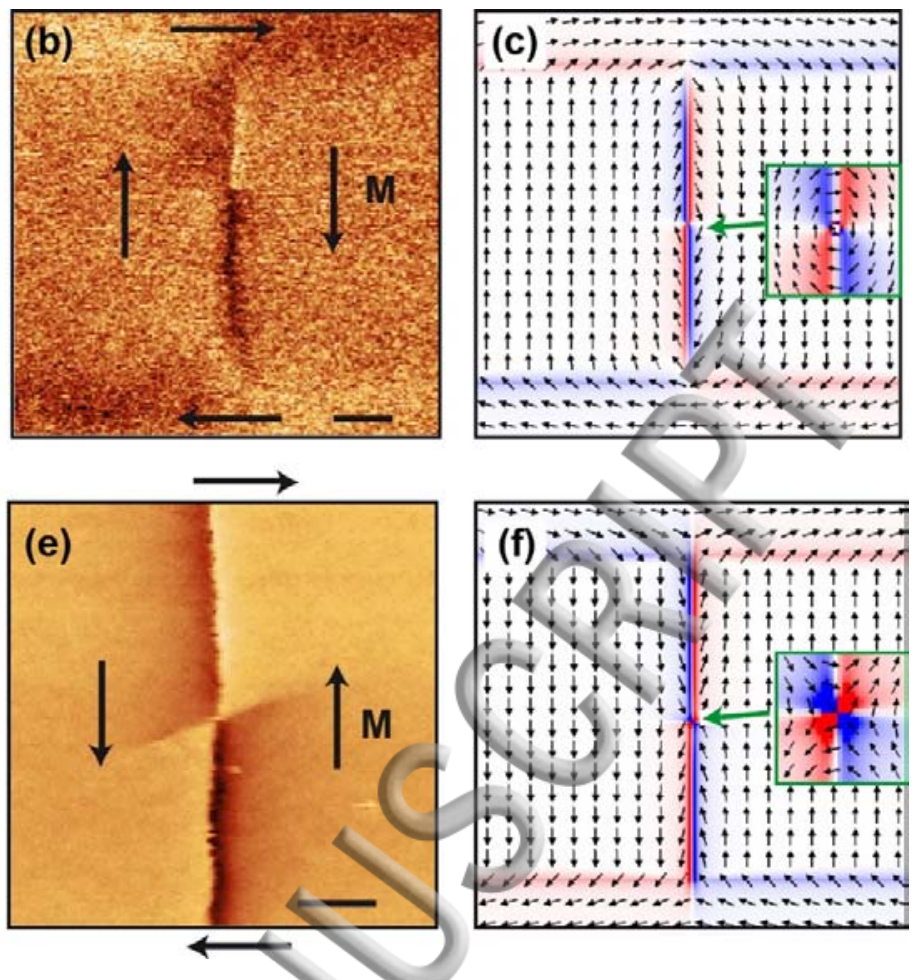

Figure 3. Stabilization of antivortex and vortex Bloch lines within patterned domain walls (a), (d) Sketches showing the geometry and direction of the patterned exchange bias for stabilizing vortex and antivortex Bloch lines, respectively. The vortex (antivortex) Bloch line is indicated by the pink (yellow) circle, and the corresponding 180 deg Néel domain wall is marked by the dashed line. The direction of the exchange bias field is indicated in blue for each patterned area. (b), (e) MFM images of the patterned structures for the yortex and antivortex Bloch line, respectively. The direction of the magnetization is indicated by the black arrows. The opposite bright-dark colors in the top and bottom parts of the domain wall confirms the change in the wall chirality, and the stabilization of the Bloch line in both cases. Scale bạrs $3 \mu \mathrm{m}$. (c), (f) Simulated micromagnetic configuration for the vortex and antivortex Bloch lines, respectively. The black arrows indicate the direction of the spins, and the red/blue color marks div (M), which is related to the MFM contrast. 
(a)
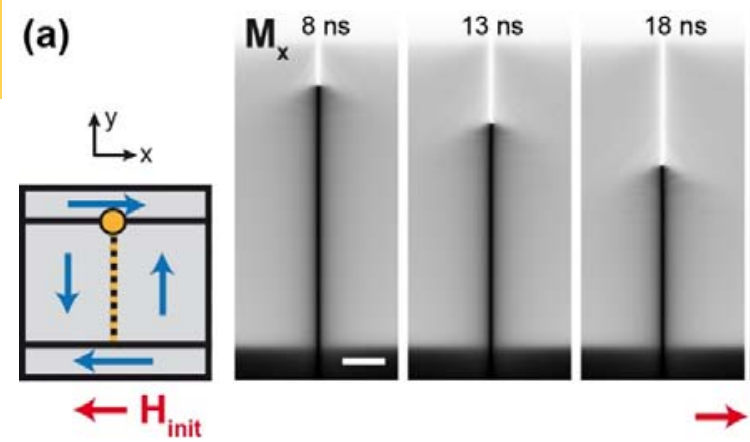

$23 \mathrm{~ns}$
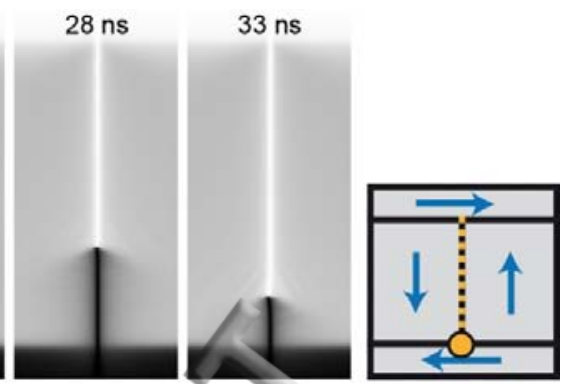

(b)
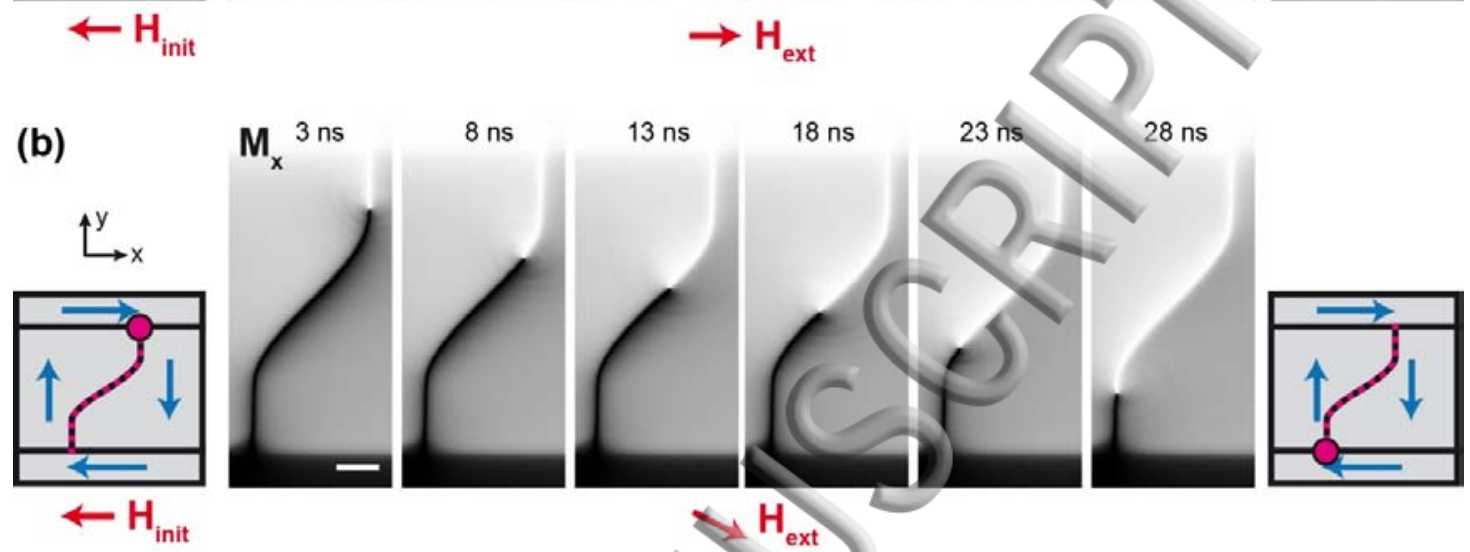

$28 \mathrm{~ns}$
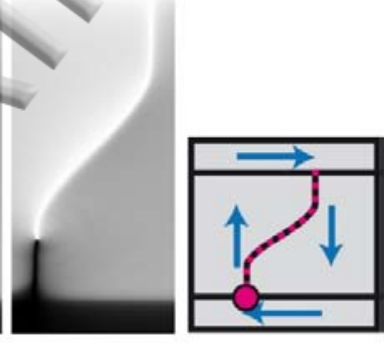

Figure 4. Manipulation of Bloch lines within nanopatterned 180 deg walls, via external fields. (a) Controlled displacement as a function of time of an antivortex Bloch line, initially stabilized at the top edge of a straight 180 deg Néel wall (see sketch on the left), via a $6.7 \mathrm{mT}$ static magnetic field applied along $+x$. (b) Displacement as a function of time of a vortex Bloch line along a zig-zag shaped 180 deg Néel wall, via a 6.7 mT static external field applied as sketched. Scale bars: $500 \mathrm{~nm}$. 

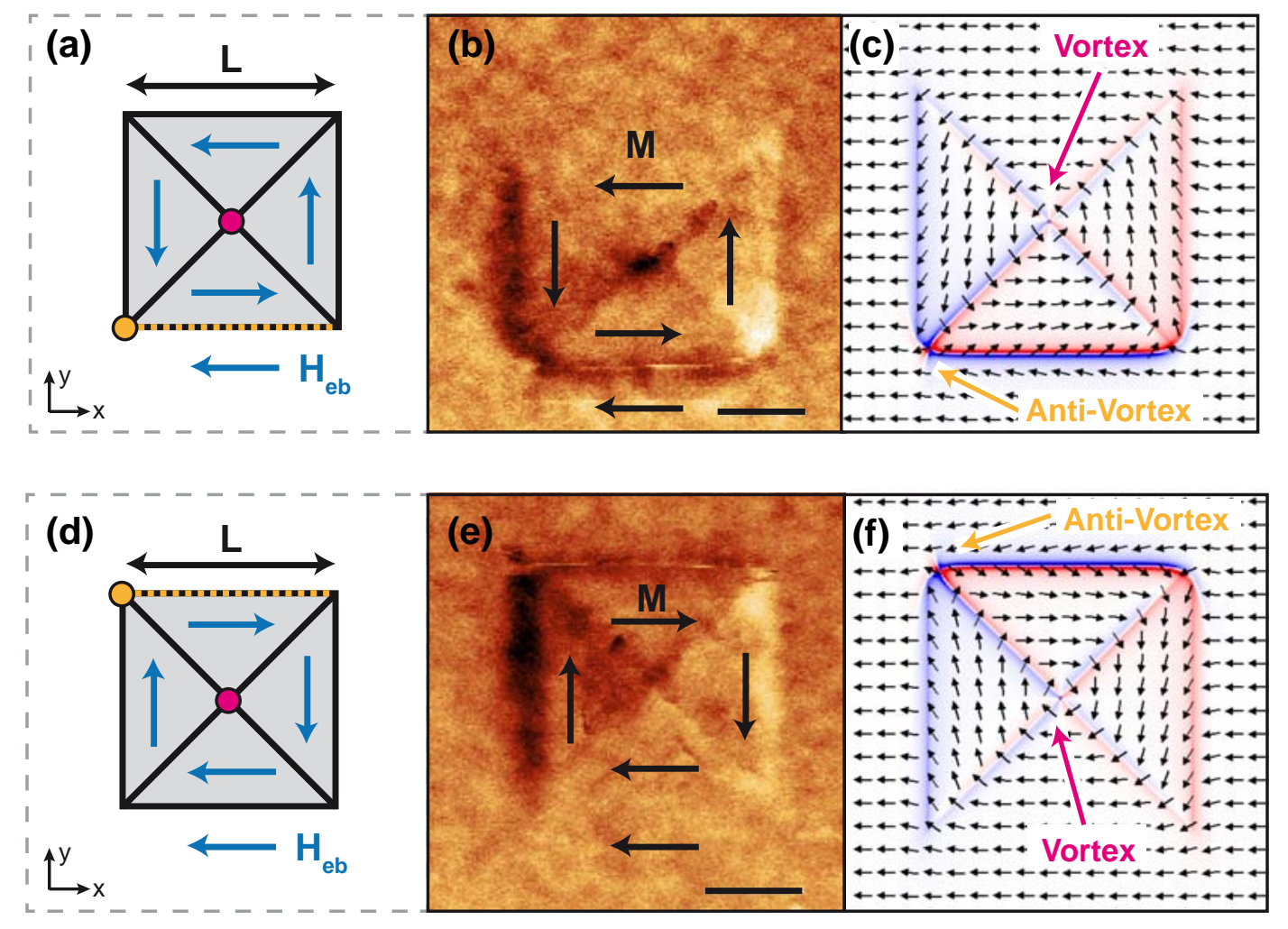


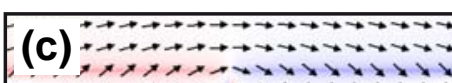

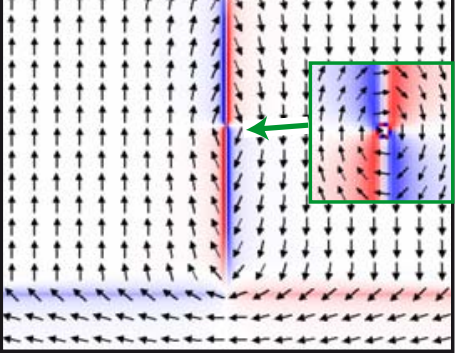

ond

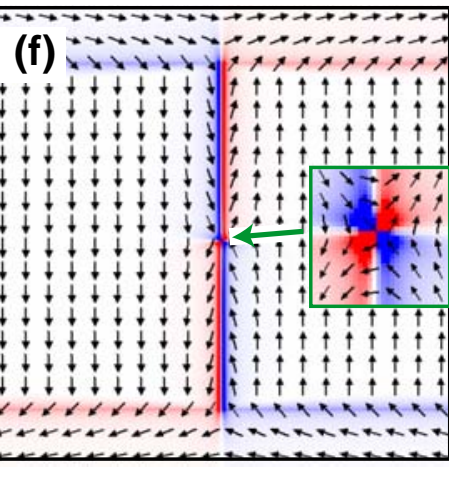

(a)

(d)

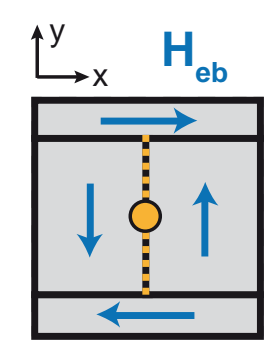

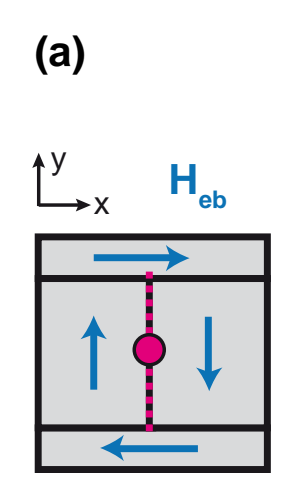

(b)

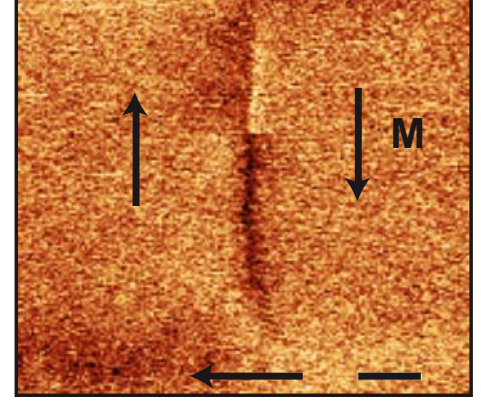

(e)

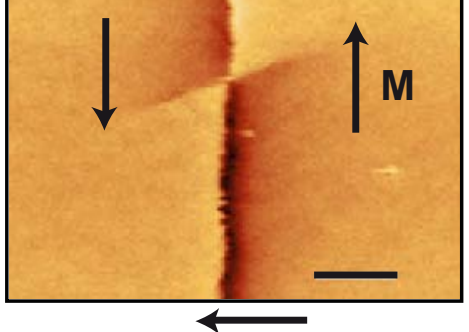


$\begin{array}{llllll}\text { (a) } & \mathbf{M}_{\mathbf{x}} 8 \mathrm{~ns} & 13 \mathrm{~ns} & 18 \mathrm{~ns} & 23 \mathrm{~ns} & 28 \mathrm{~ns}\end{array}$

$\begin{array}{lllllll}\text { (a) } & \mathbf{M}_{\mathbf{x}} 8 \mathrm{~ns} & 13 \mathrm{~ns} & 18 \mathrm{~ns} & 23 \mathrm{~ns} & 28 \mathrm{~ns} & 33 \mathrm{~ns}\end{array}$

$\begin{array}{lllllll}\text { (a) } & \mathbf{M}_{\mathbf{x}} 8 \mathrm{~ns} & 13 \mathrm{~ns} & 18 \mathrm{~ns} & 23 \mathrm{~ns} & 28 \mathrm{~ns} & 33 \mathrm{~ns}\end{array}$
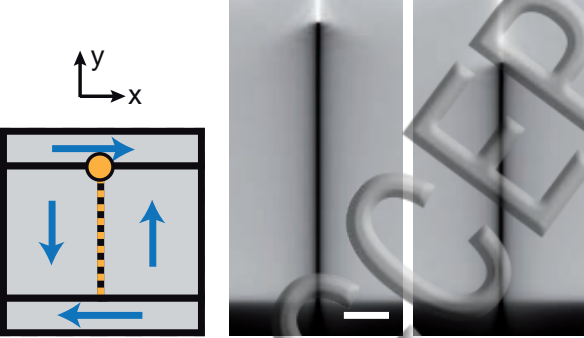

$\leftarrow \mathrm{H}_{\text {init }}$

$\rightarrow \mathrm{H}_{\text {ext }}$

(b)

$M_{x} 3$ ns

$8 \mathrm{~ns}$

$13 \mathrm{~ns}$

18 ns

23 ns

$28 \mathrm{~ns}$
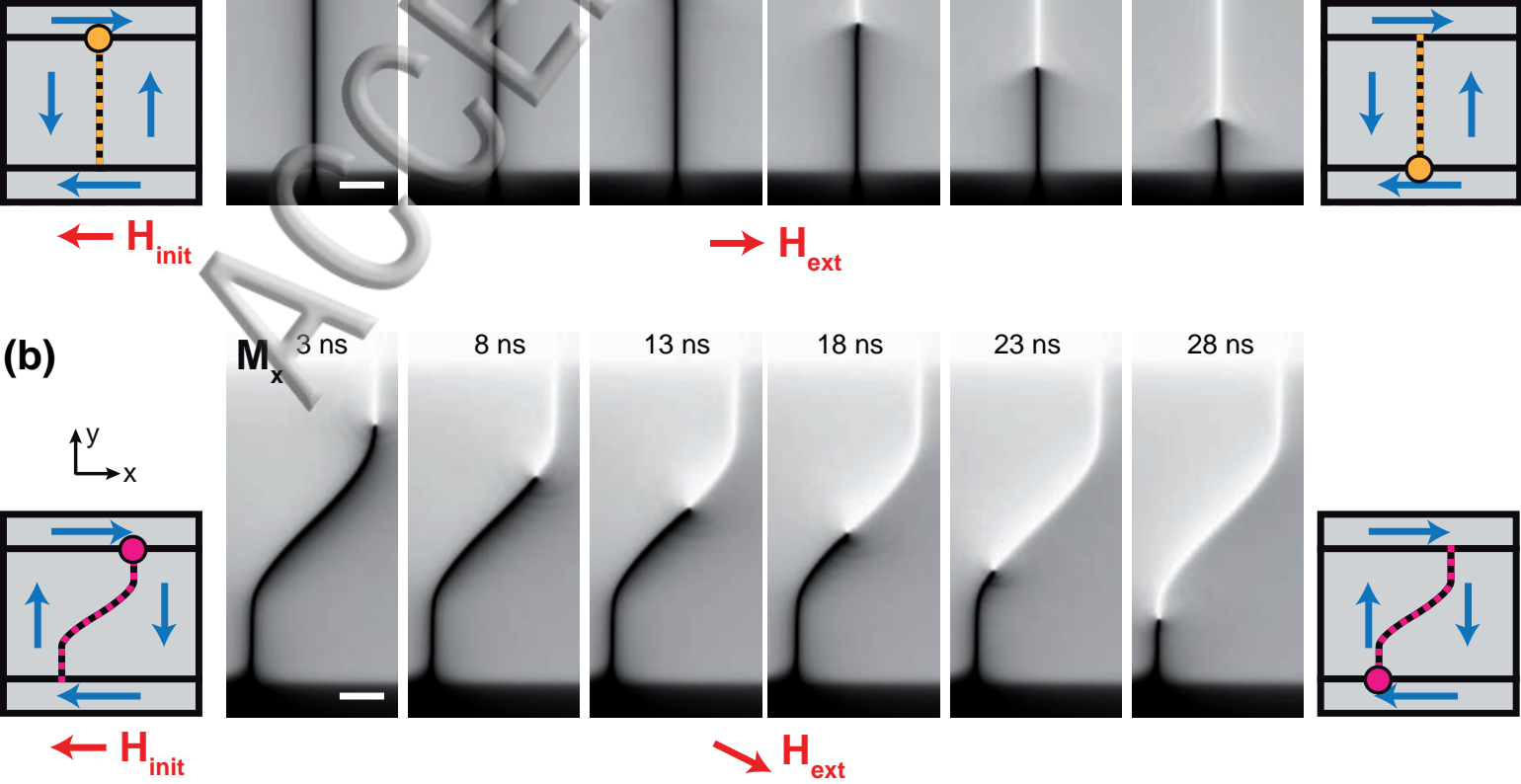

$\searrow \mathrm{H}_{\text {ext }}$
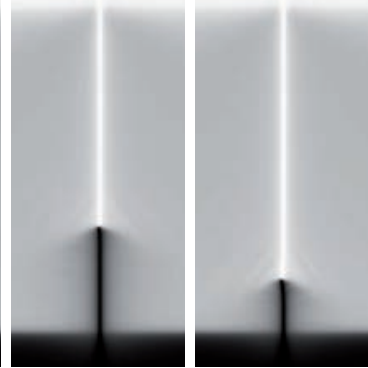

$$
\text { xt }
$$

